\title{
PREVALÊNCIA E FATORES ASSOCIADOS À POLIFARMÁCIA ENTRE IDOSOS DE UM HOSPITAL PÚBLICO
}

\author{
Vanessa da Silva Cuentro ${ }^{1}$ \\ Thayná Modesto² \\ Marcieni Ataíde de Andrade ${ }^{3}$ \\ Marcos Valerio Santos da Silva ${ }^{4}$
}

\begin{abstract}
RESUMO
A polifarmácia é um fator que tem grande impacto na segurança do paciente idoso, tendo em vista que é a grande responsável pelas interações medicamentosas. Os objetivos da presente pesquisa são identificar a prevalência e os fatores associados à polifarmácia. A metodologia utilizada foi estudo quantitativo descritivo prospectivo, baseado em análise documental por meio de um instrumento semiestruturado, analisando-se os dados no programa estatístico SPSS 20.0. Os resultados demonstraram que a média de idade foi de 71,9 anos, e 52,7\% mulheres. 0 tempo de internação obteve uma média de 21,7 dias, a média de diagnósticos por paciente foi de 2,6. 0 principal diagnóstico de internação hospitalar foi doenças do aparelho circulatório $(20,3 \%)$. A média de medicamentos prescritos por paciente foi de 6,8 . Os medicamentos mais utilizados faziam parte do sistema digestório e metabólico (32,4\%), a prevalência de prescrição de medicamentos potencialmente inadequados durante as internações avaliadas foi de $11,2 \%$. Em relação às potenciais interações medicamentosas, foram identificadas em $65,5 \%$ das prescrições e os medicamentos mais envolvidos nas interações fazem parte do sistema cardiovascular (38,6\%). Diante de tais resultados, verifica-se a necessidade de adotar estratégias para a otimização da farmacoterapia prestada ao paciente idoso.
\end{abstract}

Palavras-chave: Medicamento. Prescrição. Polifarmácia. Interações de medicamentos.

PREVALENCE AND FACTORS ASSOCIATED WITH POLYPHARMACY AMONG THE ELDERLY A PUBLIC HOSPITAL

\section{ABSTRACT}

Polypharmacy is a factor that has great impact on the security of the elderly patient, considering that is largely responsible for drug interactions. The objectives of this research were to identify the prevalence and factors associated with polypharmacy. The methodology used was a prospective descriptive quantitative study based on document analysis through a semi-structured instrument, analyzing the data in SPSS 20.0. The results demonstrated that the mean age was 71.9 years, $52.7 \%$ female. The length of stay scores a 21.7 days, the average number of diagnoses per patient was 2.6. The main hospital was diagnosed with diseases of the circulatory system (20.3\%). The average number of drugs prescribed per patient was 6.8. The most used drugs were part of the digestive and metabolic system (32.4\%), the prevalence of potentially inappropriate prescription drugs evaluated during hospitalization was $11.2 \%$. With regard to potential drug interactions, were identified in $65.5 \%$ of prescriptions drugs most involved in the interactions are part of the cardiovascular system (38.6\%). Given these results, there is the need to adopt strategies for the optimization of pharmacotherapy given to the elderly.

Keywords: Medication. Prescription. Polypharmacy. Drug Interactions.

\footnotetext{
1 Farmacêutica, Universidade Federal do Pará. vancuentro@hotmail.com

2 Farmacêutica, Universidade Federal do Pará. modesto0521@hotmail.com

3 Farmacêutica, Universidade Federal do Pará. marcieni@ufpa.br

${ }^{4}$ Farmacêutico, Universidade Federal do Pará. marcossilva@ufpa.br
} 
Polifarmácia é definida como a utilização de cinco ou mais medicamentos por dia, prática comum entre os idosos (Galvão; Ferreira, 2010). A utilização de polifarmácia associa-se com as reações adversas aos medicamentos (RAM), interações medicamentosas (IM), dificuldades na adesão ao tratamento e aumento dos custos da assistência à saúde (Costa; Pedroso, 2011).

Acrescido a esse fato têm-se particularidades fisiológicas que os idosos apresentam que levam a modificações nas propriedades farmacocinéticas e farmacodinâmicas dos medicamentos. Com o envelhecimento diminui o metabolismo hepático e a função renal. Diante dessa eliminação renal deficiente, metabólitos ativos acumulam-se e provocam toxicidade (Corsonello; Pedone; Incalzi, 2010). Sendo assim, algumas classes de medicamentos passaram a ser consideradas potencialmente inapropriadas para o idoso, pois os riscos de seu uso superam os benefícios (Chang; Chan, 2010; Holt; Schmeidl; Thurmann, 2010).

Foi elaborada, portanto, uma lista de fármacos potencialmente inapropriados para uso em idosos denominada de critérios de Beers-Fick (2012) está lista é utilizada para avaliação da qualidade da prescrição.

Logo, considerando a importância da terapia medicamentosa para pacientes idosos, uma vez que dela depende o sucesso do tratamento, o controle, a cura e a prevenção de doenças, bem como a promoção da saúde, propõe-se neste estudo identificar a prevalência e fatores associados à polifarmácia entre pacientes idosos internados em um hospital universitário.

\section{MÉTODOS}

Trata-se de um estudo transversal, observacional de caráter descritivo e analítico, a ser realizado por meio da análise dos prontuários de pacientes idosos internados nas clínicas do Hospital Universitário João de Barros Barreto (HUJBB) da Universidade Federal do Pará, no município de Belém - Pará. O
HUJBB é um hospital de nível terciário, com capacidade para 300 leitos e integrante do Sistema Único de Saúde (SUS). Atua na área de ensino, pesquisa e assistência, sendo referência no Sistema Municipal e Estadual de Saúde em pneumologia, infectologia, endocrinologia e diabetes, além de referência nacional em HIV (vírus da imunodeficiência humana).

A amostra foi composta de prontuários de pacientes idosos internados, de $1^{\circ}$ de janeiro de 2011 a 30 de janeiro de 2012, de ambos os sexos, hospitalizados na clínica médica, clínica de infectologia e clínica de pneumologia do HUJBB. A relação de pacientes internados em 2011 e 2012 foi obtida a partir do sistema de informações do censo hospitalar da instituição de saúde estudada, que constatou 776 pacientes idosos internados no período do estudo. O tamanho da amostra foi definido considerando nível de confiança de $95 \%$. Assim, o número total de pacientes foi calculado em 258. Foi atribuída uma numeração a cada prontuário, na sequência foi utilizado o método de amostragem aleatória simples sem reposição para seleção da amostra deste estudo, no programa Biostat 5.0. Inicialmente foram sorteados 258 indivíduos e para repor perdas inevitáveis a amostra original foi acrescida de 20 sujeitos.

Foram incluídos no estudo todos os pacientes com idade igual ou superior a 60 anos, com registro na Divisão de Arquivo Médico e Estatística (Dame), admitidos no período de $1^{\circ}$ de janeiro de 2011 a 30 de janeiro de 2012, com prescrição da mediana da internação para dois ou mais medicamentos. Foram excluídos do estudo pacientes com dados incompletos (sem registro de internação, nota de admissão, evolução clínica e prescrições).

A variável dependente do estudo foi a presença ou não de polifarmácia, considerando-se como polifarmácia o uso de cinco ou mais medicamentos simultaneamente (Secoli, 2010). As variáveis independentes incluíram idade, sexo, estado civil, procedência, número de diagnósticos, clínica de internação, tempo de internação, desfecho clínico, interação medicamentosa e medicamentos inapropriados. A técnica de coleta de dados foi a análise documental e consistiu na revisão retrospectiva dos 
prontuários selecionados consultando-se o registro de internação, nota de admissão, anamnese, evolução clínica do paciente e prescrições.

A caracterização demográfica e clínica dos pacientes foi composta de variáveis categóricas e variáveis quantitativas discretas. As variáveis categóricas foram sexo, estado civil, raça, procedência, clínica de internação, desfecho e diagnóstico de admissão. As variáveis quantitativas foram idade, tempo de internação (diferença entre a data de internação e a alta). Os diagnósticos de admissão foram classificados segundo o Código Internacional de Doenças - CID 10 (World..., 2008).

$\mathrm{Na}$ análise da prescrição as variáveis categóricas foram medicamento prescrito, existência de polifarmácia e prescrição de medicamentos inapropriados. A variável quantitativa foi o número de medicamentos prescritos. O nome dos medicamentos prescritos segundo o nome comercial foi identificado de acordo com a Denominação Comum Brasileira (DCB) (Brasil, 1996) e classificados de acordo com a Classificação Anatômico-Terapêutico-Clínica (ATC) proposta pela OMS até o $2^{\circ}$ nível - grupo terapêutico principal (World..., 2013); os medicamentos foram classificados como inapropriados segundo o critério de Beers-Fick. A partir do perfil de medicamentos prescritos, foram identificadas as interações medicamento-medicamento por meio da base de dados Micromedex ${ }^{\circledR}$ DrugReax System (Micromedex, 2011).

As informações coletadas foram organizadas em tabelas do banco de dados criado no software Microsoft Excel ${ }^{\circledR} 2010$. Os dados foram submetidos à análise descritiva pelo software Statistical Package for Social Sciences (SPSS) for Windows versão 20.0, para obtenção das frequências absoluta e relativa das variáveis analisadas, principalmente a polifarmácia. $O$ nível de significância adotado no estudo foi de $5 \%(\alpha=0,05)$. As análises bivariadas foram utilizadas para verificar associação entre cada uma das variáveis independentes com a variável dependente (polifarmácia), pelo teste qui-quadrado $\left(\chi^{2}\right)$.
Verificou-se a associação de variáveis quantitativas com a variável dependente expressa de forma quantitativa discreta a partir da Correlação de Pearson, sendo calculado o coeficiente de correlação entre as variáveis. As variáveis demográficas e clínicas pesquisadas foram dicotomizadas para se obter a razão de chances (OR bruta). Na análise multivariada utilizou-se a regressão de Poisson para avaliar as variáveis independentes associadas à polifarmácia, controladas por possíveis fatores de confusão (OR ajustada).

Para o estudo dos fatores associados à polifarmácia foi construído um modelo teórico de determinação com blocos hierarquizados de variáveis, as quais foram ajustadas entre si num primeiro momento. Aquelas variáveis que alcançaram um nível descritivo (p-valor) menor ou igual a 0,20 foram incluídas no modelo de regressão e ajustadas ao nível superior ao seu. Este trabalho foi aprovado pelo Comitê de Ética em Pesquisa Envolvendo Seres Humanos do Hospital Universitário João de Barros Barreto sob parecer número 45.105/2012 e CAAE02299712.0.00000.0017, de acordo com a Resolução n ${ }^{\circ}$ 466/2012 do Conselho Nacional de Saúde do Brasil.

\section{RESULTADOS E DISCUSSÃO}

$\mathrm{Na}$ amostra, composta por 258 indivíduos, houve predominância do sexo feminino $(52,7 \%)$ com pacientes idosos mais velhos que no sexo masculino ( $\mathrm{p}<0,05)$, estado civil casados $(57,8 \%)$. A maior parte desses pacientes $(67,1 \%)$ é oriunda da capital. A média de idade foi 71,9 anos, variando de 60 a 106 anos, com predominância da faixa etária de 60-69 anos $(51,6 \%)$.

Dados semelhantes também foram encontrados no estudo realizado em um hospital universitário de Minas Gerais (Rabelo et al, 2010), no qual também houve predominância da faixa etária de $60-$ 69 anos $(44,9 \%)$ e do sexo feminino (56,2\%). Esse fenômeno de feminização do envelhecimento é decorrente da maior expectativa de vida da mulher no 
Brasil, média de 77 anos (Instituto..., 2010), pois a mortalidade atinge os homens em proporção mais elevada (Giacomin, 2012).

A maior parte dos pacientes era proveniente da clínica médica (40,3\%), o tempo de internação variou de 1 a 103 dias, com média de 21,7 dias. As internações com período maior ou igual a 14 dias foram predominantes $(50,9 \%)$ e $85,7 \%$ dos pacientes receberam alta hospitalar.

Dados semelhantes foram os encontrados no estudo realizado por Motta, Hansel e Silva (2010) em um hospital do Paraná, quando o tempo predominante de internação da população do estudo foi de $85 \%$ por até 30 dias seguidos.

O paciente idoso demanda maior tempo de internação, gerando consequentemente maiores custos hospitalares, o que permite inferir que as internações da população idosa são mais onerosas do que aquelas da população adulta (Silveira, 2013).

A média de diagnósticos por paciente foi de 2,6, variando de um a cinco. Os três diagnósticos mais frequentes foram: doenças do aparelho circulatório $(20,3 \%)$, doenças do aparelho respiratório $(18,2 \%)$ e doenças infecciosas e parasitárias $(15,0 \%)$.

Em uma avaliação das informações obtidas na base de dados do Departamento de Informática do Sistema Único de Saúde do Brasil (Datasus), do Ministério da Saúde, sobre a morbidade hospitalar nos anos de 2005 e de 1994 em idosos, dentre as cinco maiores frequências encontradas de morbidade hospitalar nos idosos, a primeira enquadra-se nas doenças do aparelho circulatório (28,9\%), a segunda maior nas doenças do aparelho respiratório $(17,1 \%)$ e a quinta maior é de doenças infecciosas e parasitárias (7,19\%) (Góis; Veras, 2010).

Esses resultados corroboram com as mudanças epidemiológicas na população. Nas últimas décadas as Doenças Crônicas Não Transmissíveis (DCNT) têm liderado as causas de óbitos no país, ultrapassando as taxas de mortalidade por doenças infectocontagiosas (Carvalho et al., 2014).

$\mathrm{O}$ número de diagnósticos foi diretamente proporcional ao uso de medicamentos $(\mathrm{OR}=4,85-\mathrm{IC}$ : 2,15-10,95), concordando assim com a literatura consultada de que quanto maior o número de diagnóstico identificados, maior seria o número de medicamentos necessários (Lucchetti et al., 2010; Silva et al., 2012).

Realizando a associação entre a ocorrência de polifarmácia com variáveis demográficas observa-se que há uma tendência de ocorrência de polifarmácia mais frequente em pacientes idosos mais velhos $(p=0,07)$ (Tabela 1). Esse resultado pode ser comparado com outros estudos internacionais que demonstraram associação de polifarmácia com a idade (Nobili et al., 2011; Salih et al., 2013). Quanto ao sexo, houve maior frequência de polifarmácia em mulheres $(52,7 \%)$, porém não se observou diferença estatística significativa. O mesmo ocorreu em relação ao estado civil e à procedência dos indivíduos, respectivamente com $57,8 \%$ e de $67,1 \%$ pacientes idosos submetidos à polifarmácia.

Tabela 1 - Associação da ocorrência de polifarmácia com variáveis sociodemográficas - Belém, 2013

\begin{tabular}{|c|c|c|c|c|c|c|c|}
\hline \multirow{3}{*}{ Variáveis } & \multicolumn{4}{|c|}{ Polifarmácia } & \multirow{2}{*}{\multicolumn{2}{|c|}{ Total }} & \multirow{3}{*}{$\mathbf{p}^{*}$} \\
\hline & \multicolumn{2}{|c|}{ Sim } & \multicolumn{2}{|c|}{ Não } & & & \\
\hline & Fa & fr( $(\%)$ & Fa & fr(\%) & $\mathbf{F a}$ & $\operatorname{fr}(\%)$ & \\
\hline \multicolumn{8}{|l|}{ Idade } \\
\hline$\geq 70$ anos & 107 & 51,2 & 18 & 36,7 & 125 & 48,4 & 0,07 \\
\hline$<70$ anos & 102 & 48,8 & 31 & 63,3 & 133 & 51,6 & \\
\hline \multicolumn{8}{|l|}{ Sexo } \\
\hline Feminino & 108 & 51,7 & 28 & 57,1 & 136 & 52,7 & 0,49 \\
\hline Masculino & 101 & 48,3 & 21 & 42,9 & 122 & 47,3 & \\
\hline \multicolumn{8}{|l|}{ Estado Civil } \\
\hline Casado(a) & 120 & 57,4 & 29 & 59,2 & 149 & 57,8 & 0,82 \\
\hline Solteiro(a)/ & & & & & & & \\
\hline Viúvo(a) & 89 & 20,1 & 20 & 18,4 & 51 & 19,8 & \\
\hline \multicolumn{8}{|l|}{ Procedência } \\
\hline Capital & 144 & 68,9 & 29 & 59,2 & 173 & 67,1 & 0,19 \\
\hline Interior & 65 & 31,1 & 20 & 40,8 & 85 & 32,9 & \\
\hline Total & 209 & 100,0 & 49 & 100,0 & 258 & 100,0 & ----- \\
\hline
\end{tabular}

Ao se verificar o número de medicamentos prescritos obteve-se uma variação de 2 a 16, em um total de 1.762 especialidades farmacêuticas; verificou-se que as mulheres utilizaram em média 6,8 , semelhante à média dos homens, que foi de 6,9 medicamentos. A média por paciente foi de 6,8 medicamentos: $9(3,5 \%)$ idosos utilizavam dois medicamentos; 11 (4,3\%) utilizavam três; 30 (11,6\%) 
utilizavam quatro medicamentos e $208(80,6 \%)$ idosos faziam o uso de cinco ou mais medicamentos, caracterizando a polifarmácia.

Estes resultados coincidiram com um estudo realizado por Iniesta-Navalón, Urbieta-Sanz e Gascón-Cánovas (2011) que registrou uma média de 7,6 medicamentos por paciente, com 78,8\% dos pacientes com polifarmácia.

Na Tabela 2 observa-se que ocorreu maior frequência da polifarmácia quanto ao maior número de diagnósticos $(51,2 \%)$ e ao maior tempo de internação $(54,1 \%)$, destacando-se associação estatística da ocorrência da polifarmácia com o número de diagnósticos e o tempo de internação hospitalar. Não houve associação estatística com a clínica em que o indivíduo foi internado. Quanto ao desfecho clínico, $16,3 \%$ dos pacientes idosos com polifarmácia foram a óbito, o que pode indicar uma tendência de associação clínica entre as duas ocorrências, apesar da ausência de associação estatística $(\mathrm{p}=0,07)$.

Tabela 2 - Associação da ocorrência de polifarmácia com variáveis clínicas - Belém, 2013

\begin{tabular}{|c|c|c|c|c|c|c|c|}
\hline \multirow{3}{*}{ Variáveis } & \multicolumn{4}{|c|}{ Polifarmácia } & \multirow{2}{*}{\multicolumn{2}{|c|}{ Total }} & \multirow{3}{*}{ p* } \\
\hline & \multicolumn{2}{|c|}{ Sim } & \multicolumn{2}{|c|}{ Não } & & & \\
\hline & $\mathbf{F a}$ & $\operatorname{fr}(\%)$ & $\mathbf{F a}$ & fr(\%) & $\mathbf{F a}$ & $\operatorname{fr}(\%)$ & \\
\hline \multicolumn{8}{|l|}{$\begin{array}{l}\text { Número de } \\
\text { diagnósticos }\end{array}$} \\
\hline $\begin{array}{c}\geq 3 \\
\text { diagnósticos } \\
<3\end{array}$ & 107 & 51,2 & 8 & 16,3 & 115 & 44,6 & $<0,01$ \\
\hline diagnósticos & 102 & 48,8 & 41 & 83,7 & 143 & 55,4 & \\
\hline \multicolumn{8}{|l|}{ Clínica } \\
\hline DIP & 60 & 28,7 & 16 & 32,7 & 76 & 29,5 & 0,44 \\
\hline Médica & 89 & 42,6 & 16 & 32,7 & 105 & 40,7 & \\
\hline Pneumologia & 60 & 28,7 & 17 & 34,7 & 77 & 29,8 & \\
\hline \multicolumn{8}{|l|}{$\begin{array}{ll}\text { Tempo de } \\
\text { Internação }\end{array}$} \\
\hline$\geq 14$ dias & 113 & 54,1 & 16 & 32,7 & 129 & 50,0 & $<0,01$ \\
\hline$<14$ dias & 96 & 45,9 & 33 & 67,3 & 129 & 50,0 & \\
\hline \multicolumn{8}{|l|}{$\begin{array}{l}\text { D e s f e c ho } \\
\text { clínico }\end{array}$} \\
\hline Óbito & 34 & 16,3 & 3 & 6,1 & 37 & 14,3 & 0,07 \\
\hline Alta & 175 & 83,7 & 46 & 93,9 & 221 & 85,7 & \\
\hline Total & 209 & 100,0 & 49 & 100,0 & 258 & 100,0 & -- \\
\hline
\end{tabular}

*Teste do Qui-quadrado. DIP: Clínica de doenças infecciosas e parasitárias. fa: frequência absoluta; fr (\%): frequência relativa em percentual.

Fonte: Dados dos Autores.
Na Tabela 3 observa-se associação da ocorrência da polifarmácia com a de interação medicamentosa e de medicamento inapropriado $(p<0,01)$. A frequência de pacientes idosos submetidos à polifarmácia que apresentaram interação medicamentosa e medicamento inapropriado foi respectivamente de $76,6 \%$ e $58,4 \%$.

Um estudo de corte transversal entre os meses de janeiro a dezembro de 2007, com registros de pacientes com idade acima de 60 anos internados no Hospital Geral de Vitória da Conquista, conclui que o uso de medicamento impróprio está associado à polifarmácia $(\mathrm{p}<0,01)$ (Guimarães; Moura, 2012).

Verifica-se que o número elevado de fármacos prescritos a esses pacientes está associado ao risco do emprego de medicamentos inapropriados, o que eleva o risco de RAM em indivíduos idosos, grupo de pacientes muito vulneráveis, em virtude de suas particularidades farmacocinéticas e farmacodinâmicas (Secoli, 2010).

Tabela 3 - Associação da ocorrência de Polifarmácia com variáveis sobre prescrição medicamentosa - Belém, 2013

\begin{tabular}{|c|c|c|c|c|c|c|c|}
\hline \multirow{3}{*}{ Variáveis } & \multicolumn{4}{|c|}{ Polifarmácia } & \multirow{2}{*}{\multicolumn{2}{|c|}{ Total }} & \multirow{3}{*}{$\mathbf{p}^{*}$} \\
\hline & \multicolumn{2}{|c|}{ Sim } & \multicolumn{2}{|c|}{ Não } & & & \\
\hline & $\mathbf{F a}$ & fr( $(\%)$ & $\mathbf{F a}$ & $\operatorname{fr}(\%)$ & $\mathbf{F a}$ & $\operatorname{fr}(\%)$ & \\
\hline \multicolumn{8}{|c|}{$\begin{array}{l}\text { I n t e r a ç ã o } \\
\text { medicamentosa }\end{array}$} \\
\hline Sim & 160 & 76,6 & 9 & 18,4 & 169 & 65,5 & $<0,01$ \\
\hline Não & 49 & 23,4 & 40 & 81,6 & 89 & 34,5 & \\
\hline \multicolumn{8}{|c|}{$\begin{array}{l}\text { Medicamento } \\
\text { inapropriado }\end{array}$} \\
\hline Sim & 122 & 58,4 & 13 & 26,5 & 135 & 52,3 & $<0,01$ \\
\hline Não & 87 & 41,6 & 36 & 73,5 & 123 & 47,7 & \\
\hline Total & 209 & 100,0 & 49 & 100,0 & 258 & 100,0 & ---- \\
\hline
\end{tabular}

Não houve ocorrência de correlação entre a polifarmácia e a idade dos pacientes idosos; entretanto observou-se correlação positiva regular $(0,3 \leq$ $\mathrm{r}<0,6)$ com o tempo de internação, o número de diagnósticos e a quantidade de medicamentos inapropriados, havendo correlação positiva forte $(0,6 \leq$ $r<0,9)$ entre polifarmácia nos pacientes idosos internados e o número de interações medicamentosas. 
Em um estudo realizado por Leão, Moura e Medeiros (2014) em que se avaliou prescrições dispensadas em Unidades de Saúde em Vitória da Conquista - BA, mostrou em teste qui-quadrado associação positiva entre polifarmácia e ocorrência de interações medicamentosas potenciais $(\mathrm{p}<$ 0,001), o que deixa claro a necessidade de controle farmacoterapêutico mais rigoroso.

Nas Tabelas 4 e 5 são apresentadas as análises bivariada e multivariada dos blocos de variáveis relacionadas aos fatores demográficos e clínicos.

$\mathrm{Na}$ análise bruta, no bloco de variáveis demográficas, a mais associada à ocorrência de polifarmácia foi "idade", ainda que não tenha sido uma associação significativa. As variáveis clínicas "número de diagnósticos" e "tempo de internação" estavam mais associadas à polifarmácia, com a variável "desfecho" apresentando menor associação e sem mostrar associação significativa (Tabela 4).

Tabela 4 - Ocorrência de polifarmácia segundo variáveis sociodemográficas e clínicas - Belém, 2013

\begin{tabular}{|c|c|c|c|c|}
\hline \multirow[t]{2}{*}{ Variável } & \multirow{2}{*}{$\begin{array}{c}\text { OR bruta } \\
\left(\mathrm{IC}_{95 \%}\right)\end{array}$} & \multicolumn{3}{|c|}{ OR ajustada } \\
\hline & & $\mathbf{P}$ & $\left(\mathrm{IC}_{95 \%}\right)$ & $\mathbf{P}$ \\
\hline \multicolumn{5}{|c|}{$1^{\circ}$ Bloco: Polifarmácia e variáveis demográficas } \\
\hline Idade & & 0,07 & & 0,05 \\
\hline$\geq 70$ anos & $1,81(0,95-3,43)$ & & $1,95(1,01-3,76)$ & \\
\hline$<70$ anos & 1 & & & \\
\hline Sexo & & 0,49 & & 0,28 \\
\hline Feminino & $0,80(0,43-1,50)$ & & $0,70(0,37-1,33)$ & \\
\hline Masculino & 1 & & 1 & \\
\hline \multicolumn{5}{|c|}{$2^{\circ}$ Bloco: Polifarmácia e variáveis clínicas } \\
\hline $\begin{array}{l}\text { Número de } \\
\text { diagnósticos }\end{array}$ & & $<0,01$ & & $<0,01$ \\
\hline$\geq 3$ diagnósticos & $5,38(2,40-12,02)$ & & $4,53(2,00-10,29)$ & \\
\hline$<3$ diagnósticos & 1 & & 1 & \\
\hline $\begin{array}{l}\text { Tempo de } \\
\text { Internação }\end{array}$ & & $<0,01$ & & 0,03 \\
\hline$\geq 14$ dias & $2,43(1,26-4,68)$ & & $2,17(1,09-4,29)$ & \\
\hline$<14$ dias & 1 & & 1 & \\
\hline Desfecho & & 0,05 & & 0,20 \\
\hline Óbito & $2,98(0,88-10,13)$ & & $2,31(0,64-8,25)$ & \\
\hline Alta & 1 & & 1 & \\
\hline
\end{tabular}

OR bruta: Razão de chances - Análise bivariada. IC95\%: Intervalo de Confiança de $95 \%$. p: nível descritivo do teste de associação $\chi^{2}$ (Quiquadrado). OR ajustada: variáveis ajustadas entre si - Análise múltipla.

Fonte: Dados dos autores.

A análise ajustada entre os blocos destacou como determinantes na ocorrência de polifarmácia entre os pacientes idosos internados as variá- veis clínicas "número de diagnósticos" $(4,85$ vezes maior ocorrência de polifarmácia em pacientes idosos com três ou mais diagnósticos de patologias) e "tempo de internação" (2,06 vezes maior ocorrência em pacientes idosos com 14 ou mais dias de internação) (Tabela 5).

Tabela 5 - Resultados da análise de regressão multivariada em blocos hierarquizados para a ocorrência de polifarmácia nos pacientes idosos - Belém, 2013

\begin{tabular}{|c|c|c|c|c|}
\hline \multirow[t]{2}{*}{ Variável } & \multirow{2}{*}{$\begin{array}{c}\text { OR bruta } \\
\left(\mathrm{IC}_{95 \%}\right)\end{array}$} & \multicolumn{3}{|c|}{ OR ajustada } \\
\hline & & $\mathbf{P}$ & $\left(\mathrm{IC}_{95 \%}\right)$ & $\mathbf{P}$ \\
\hline \multicolumn{5}{|c|}{$1^{\circ}$ Bloco: Polifarmácia e variáveis demográficas } \\
\hline Idade & & 0,07 & & 0,12 \\
\hline$\geq 70$ anos & $1,81(0,95-3,43)$ & & $1,68(0,86-3,29)$ & \\
\hline$<70$ anos & 1 & & 1 & \\
\hline \multicolumn{5}{|c|}{$2^{\circ}$ Bloco: Polifarmácia e variáveis clínicas ${ }^{\mathrm{a}}$} \\
\hline $\begin{array}{ll}\text { Número } & \text { de } \\
\text { diagnósticos } & \end{array}$ & & $<0,01$ & & $<0,01$ \\
\hline$\geq 3$ diagnósticos & $5,38(2,40-12,02)$ & & $4,85(2,15-10,95)$ & \\
\hline$<3$ diagnósticos & 1 & & 1 & \\
\hline $\begin{array}{l}\text { Tempo de } \\
\text { Internação }\end{array}$ & & $<0,01$ & & 0,04 \\
\hline$\geq 14$ dias & $2,43(1,26-4,68)$ & & $2,06(1,04-4,08)$ & \\
\hline$<14$ dias & 1 & & 1 & \\
\hline
\end{tabular}

OR bruta: Razão de chances - Análise bivariada. IC95\%: Intervalo de Confiança de 95\%. p: nível descritivo do teste de associação $\chi^{2}$ (Quiquadrado). OR ajustada: variáveis ajustadas entre si - Análise múltipla. ${ }^{\mathrm{a}}$ Variáveis ajustada entre si e pelo $1^{\mathrm{o}}$ Bloco. ${ }^{\mathrm{b}}$ Variáveis ajustada entre si e pelos $1^{\circ}$ e $2^{\circ}$ Blocos.

Fonte: Dados dos autores.

Os resultados do presente estudo comprovaram que polifarmácia parece contribuir para o aumento da ocorrência de prescrição de medicamentos inapropriados (Nassur et al., 2010) e potenciais interações medicamentosas (Melgaço et al., 2011), fatores diretamente associados com eventos adversos relacionados à idade.

Assim sendo, nota-se que existe uma espécie de "círculo vicioso" que envolve a polifarmácia com o idoso, pois o envelhecimento traz consigo maior número de doenças, risco aumentado de internação hospitalar, regime terapêutico complexo com excesso de medicamentos prescritos, prescrição de medicamentos inapropriados e potenciais interações medicamentosas que estão fortemente associados à ocorrência de eventos adversos Além disso, os sin- 
tomas dos eventos adversos podem ser confundidos com uma nova doença, podendo resultar em nova prescrição médica.

\section{CONCLUSÃ̃o}

Verificou-se, no presente estudo, que a ocorrência da polifarmácia é de etiologia multifatorial, podendo originar-se da combinação de vários fatores, tais como pacientes idosos mais velhos, número de diagnósticos, tempo de internação hospitalar, interação medicamentosa e a ocorrência de medicamento inapropriado. Apresentam-se como principais elementos associados à polifarmácia: potenciais interações medicamentosas e medicamentos inapropriados, fatores estes que levam a eventos adversos frequentes em idosos que podem prolongar o tempo de internação hospitalar e aumentar significativamente o perfil de morbimortalidade desta faixa etária.

Eis, portanto, o grande desafio enfrentado pelos profissionais de saúde de um país que, como o Brasil, está envelhecendo: contribuir para a promoção do uso racional dos medicamentos. Com a educação apropriada desses profissionais, algumas estratégias podem auxiliar uma adequada prescrição geriátrica, por exemplo: a substituição ou inclusão de medicamentos; o aprazamento criterioso dos horários da prescrição médica, de modo que se evite a administração simultânea de medicamentos que podem interagir entre si e, por fim, a monitoração dos eventos adversos. Isso será capaz de melhorar a qualidade do serviço prestado e reduzir a incidência dos eventos adversos causados pela polifarmácia.

Finalmente, deve ser feito um esforço para limitar e racionalizar a prescrição de medicamentos. Para alcançar esse objetivo é necessário que o médico, juntamente com o apoio do farmacêutico clínico, esteja ciente da dinâmica da polifarmácia na prática clínica atual, adequando a prescrição de medicamentos com análise não só de qual medicamento utilizar, como também na quantificação de medicamentos, considerando obviamente as circunstâncias clínicas individuais de cada paciente.

\section{AGRADECIMENTOS}

À Coordenação de Aperfeiçoamento de Pessoal de Nível Superior (Capes) pela concessão da bolsa de Mestrado e ao Hospital Universitário João de Barros Barreto pela oportunidade de realização deste estudo.

\section{CONFLITO DE INTERESSE}

Declaramos não ter conflito de interesses em relação ao presente texto.

\section{REFERÊNCIAS}

AMERICAN GERIATRICS SOCIETY. Beers Criteria Update Expert Panel. American Geriatrics Society updated Beers Criteria for potentially inappropriate medication use in older adults. $J$. Am. Geriatr. Soc., v. 60, p. 616-631, 2012.

BRASIL. Lei $\mathrm{n}^{\circ} .8842 / 94$. Regulamentada pelo decreto ${ }^{\circ} 1948$ de 4 de janeiro de 1996. Estabelece a política nacional do idoso. Diário Oficial da União, Brasília, 1996. Disponível em: <http://www.planalto.gov.br/ccivil_03/leis/18842.htm>. Acesso em: 5 jan. 2013).

CARVALHO, M. H. R. D. et al . Tendência de mortalidade de idosos por doenças crônicas no município de Marília-SP, Brasil: 1998 a 2000 e 2005 a 2007. Epidemiol. Serv. Saúde, Brasília, v. 23, n. 2, jun. 2014.

CHANG, C. B.; CHAN, D. C. Comparison of published explicit criteria for potentially inappropriate medications in older adults. Drugs Aging, v. 27, p. 947-957, 2010.

COSTA, S. C.; PEDROSO, E. R. P. A prescrição de medicamentos para idosos internados em serviço de clínica médica: atualização. Rev Med Minas Gerais, v. 21, n. 2, p. 201-214, 2011.

CORSONELLO, A.; PEDONE, C.; INCALZI, R. A. Agerelated pharmacokinetic and pharmacodynamic changes and related risk of adverse drug reactions. Curr. Med. Chem., v. 17, n. 6, p. 571-584, 2010. 
COSTA, S. C.; PEDROSO, Ê. R. P. A prescrição de medicamentos para idosos internados em serviço de clínica médica: atualização. Rev. Med. Minas Gerais, v. 21, n. 2, p. 201-214, 2011.

GALVÃO, M. P. A; FERREIRA, M. B. C. Prescrição de medicamentos em geriatria. In: FUCHS, F. D.; WANNMACHER, L.; FERREIRA, M. B. C. Farmacologia clínica: fundamentos da terapêutica racional. 4. ed. Rio de Janeiro: Guanabara Koogan, 2010. p. 1.282.

GIACOMIN, K. C. Envelhecimento populacional e os desafios para as políticas públicas. In: BERZINS, M. V;; BORGES, M. C. (Orgs.). Politicas públicas para um país que envelhece. São Paulo: Martinari, 2012. p. $17-44$.

GÓIS, A. L. B. D.; VERAS, R. P. Informações sobre a morbidade hospitalar em idosos nas internações do Sistema Único de Saúde do Brasil. Ciênc. saúde coletiva, Rio de Janeiro, v. 15, n. 6, p. 2.8592.869, 2010.

GUIMARÃES, P. L; MOURA, C. S. Fatores associados ao uso de medicamentos impróprios de alto risco em pacientes idosos hospitalizados. Rev. Bras. Farm. Hosp. Serv. Saúde, v. 3, n. 4, p. 15-19, 2012.

HOLT, S.; SCHMEIDL, S; THURMANN, P. A. Potentially inappropriate medications in the elderly: the Priscus List. Dtsch. Arztebl. Int., v. 107, 2010. p. $5430-551$.

INSTITUTO BRASILEIRO DE GEOGRAFIA E ESTATÍSTICA. IBGE. Sintese de Indicadores Sociais: uma análise das condições de vida da população brasileira. Rio de Janeiro, 2010. IBGE, 2010.

INIESTA-NAVALÓN, C; URBIETA-SANZ, E; GASCÓN-CÁNOVAS, J. J. Análisis de las interacciones asociadas a la farmacoterapia domiciliaria en pacientes ancianos hospitalizados. Rev. Clin. Esp., v. 211, n. 7, p. 344-351, 2011.

LEÃO, D. F. L.; MOURA, C. S.; MEDEIROS, D. S. Avaliação de interações medicamentosas potenciais em prescrições da atenção primária de Vitoria da Conquista (BA), Brasil. Ciênc. saúde coletiva, Rio de Janeiro, v. 19, n. 1, p. 311-318, 2014.

LUCCHETTI, G. et al. Fatores associados à polifarmácia em idosos institucionalizados. Rev. Bras. Geriatr. Gerontol., v. 13, n. 1, p. 51-58, 2010.

MELGAÇO, T. B. et al. Polifarmácias e ocorrências de possíveis interações medicamentosas. Rev. Para. Med., v. 25, n. 1, 2011.
MICROMEDEX(R) HEALTHCARE SERIES [database on the Internet]. Versão 5.1. Greenwood Village, Colo: Thomson Micromedex, 2011.

MOTTA, C. C. R. D; HANSEL, C. G; SILVA, J. D. Perfil de internações de pessoas idosas em um hospital público. Rev. Eletr. Enf., v. 12, n. 3, p. 471477, 2010.

NASSUR, B. A. et al. Avaliação dos medicamentos inapropriados utilizados por idosos admitidos em hospital geral filantrópico. Rev. Bras. Clin. Med., São Paulo, v. 8, n. 3, p. 208-211, 2010.

NOBILI, A. et al. Polypharmacy, length of hospital stay, and in-hospital mortality among elderly patients in internal medicine wards. The Reposi study. Eur J Clin Pharmacol., v. 67, n. 5, p. 507519, 2011.

RABELO, L. P. D. O. et al. Perfil de idosos internados em um hospital universitário. Reme Rev. Min. Enferm., v. 14, n. 3, p. 293-300, 2010.

SALIH, S. B. et al. Prevalence and associated factors of polypharmacy among adult Saudi medical outpatients at a tertiary care center. J. Family. Community. Med., v. 20, n. 3, p. 162-167, 2013.

SECOLI, S. R. Polifarmácia: interações e reações adversas no uso de medicamentos por idosos. Rev. Bras.Eenferm., Brasília., v. 63, n. 1, p. 136-140, 2010.

SILVA, G. D. O. B. et al. Uso de medicamentos contínuos e fatores associados em idosos de Quixadá, Ceará. Rev. Bras. Epidemiol., São Paulo, v. 15, n. 2, p. 386-395, 2012.

SILVEIRA, R. E. et al. Gastos relacionados a hospitalizações de idosos no Brasil: perspectivas de uma década. Einstein., v. 11, n. 4, p. 514-520, 2013.

World Health Organization. Tradução do centro colaborador da OMS para a classificação de doenças em português (CBCD). Classificação estatística internacional de doenças e problemas relacionados à saúde-CID10, 2008. Disponível em: <http://www. datasus.gov.br/cid10/v2008/cid10.htm>. Acesso em: 5 jan. 2013.

. ATC index with DDD's, 2013. Disponível em: $<$ http://www.whocc.no/atc_ddd_index $>$. Acesso em: 5 jan. 2013.

Recebido em: 18/7/2015

Aceito em: 29/2/2016 